\title{
WI - Call for Papers Heft 3/2014
}

\section{Decision Analytics}

DOI 10.1007/s11576-012-0344-0

\section{Die Autoren

Prof. Dr. Leena Suhl ( $₫)$
DS\&OR Lab
Universität Paderborn
Warburger Straße 100
33098 Paderborn
Deutschland
suhl@dsor.de

Prof. Dr. Stefan Voß

Institut für Wirtschaftsinformatik

Universität Hamburg

Von-Melle-Park 5

20146 Hamburg

Deutschland

stefan.voss@uni-hamburg.de

Online publiziert: 2013-01-04

This article is also available in English via http://www.springerlink.com and http://www.bise-journal.org: Suhl L, Voß S (2012) BISE - Call for Papers Issue $3 / 2014$. Decision Analytics. Bus Inf Syst Eng. doi: 10.1007/ s12599-012-0242-4.

(C) Springer Fachmedien Wiesbaden 2012

\section{Schwerpunktthema}

Heutzutage erlauben moderne Informations- und Kommunikationssysteme (IKS) es, mit großen Mengen an digitalen, strukturierten Daten die Qualität computergestützter Entscheidungsvorbereitung und -unterstützung zu verbessern. Die Möglichkeiten sowohl von Hard- als auch Software haben sich im Laufe der Jahre kontinuierlich verbessert. IKS bieten Wettbewerbsvorteile in verschiedenen Bereichen, wie zum Beispiel Supply-Chain-Management, Logistik, Marketing und Telekommunikation. Moderne IKS stellen wichtige Komponenten bei der Entscheidungsunterstützung dar und basieren insbesondere auf effizienten analytischen Methoden. Simulation und Optimierung können zum Beispiel bei Fragestellungen der Preisgestaltung, der Beschaffung, der Planung sowie in vielen anderen Bereichen eingesetzt werden. Effiziente Algorithmen kombinieren sowohl Heuristiken als auch Metaheuristiken mit exakten Methoden. Neben diesen Algorithmen wurden auch im Bereich anspruchsvoller Analyse-Tools maßgebliche Fortschritte erzielt und diese erlauben den effizienten Umgang mit großen Datenmengen. Intelligente Geräte ermöglichen die Kommunikation mit verschiedenen Partnern, um den Zugang zu relevanten Daten, das Sammeln und den Austausch von Produktinformationen, sowie das Verhandeln von Preisen zu erleichtern.

Unter Decision Analytics verstehen wir die Theorie, Methoden und Praxis der Analyse und Unterstützung von Entscheidungen in Unternehmen und Organisationen mit Hilfe von formalen Modellen und Methoden. $\mathrm{Zu}$ den eingesetzten Technologien gehören die Optimierung und Methoden mit einer oder mehreren Zielfunktionen, Entscheidungsunterstützung unter Unsicherheit, die Risikoanalyse und -bewertung, die Unterstützung von Gruppenentscheidungen sowie Entscheidungsunterstützungs- und Expertensysteme. Es werden Data-Mining, Optimierung, Simulation, Mustererkennung, präskriptive bzw. prädiktive Verfahren sowie Performance-Management betrachtet. Durch geeignete Werkzeuge können relevante Entscheidungsaspekte modelliert und bewertet werden, sodass wichtige Erkenntnisse zur Entscheidungsfindung herausgearbeitet werden können. Anwendungen umfassen sowohl strategische als auch taktische und operative Entscheidungsbereiche, unter anderem in den Bereichen Supply-Chain-Management, Logistik, Produktionssysteme, Transport, Scheduling, Marketing, Personalwesen und Telekommunikation.
Das Schwerpunktheft strebt an, Synergien zwischen Decision Analytics und Wirtschaftsinformatik herzustellen. Es dient als ein Forum für Praktiker und Wissenschaftler, um hochqualitative originäre Forschungsergebnisse und Fallbeispiele in einem hochrangigen Journal $\mathrm{zu}$ veröffentlichen. Thematisch relevant sind unter anderem die folgenden Bereiche insbesondere in Kombination mit betrieblichen Anwendungen:

- Angewandte Wahrscheinlichkeitstheorie und stochastische Prozesse

- Business Intelligence

- Business Simulation

- Data-Mining und Knowledge Discovery

- Entscheidungsunterstützungssysteme

- Prognose

- Entscheidungsunterstützung mit Fuzzy-Technologien

- Gruppenbasierte Entscheidungsunterstützung

- Heuristiken, Metaheuristiken und hybride Verfahren

- Wissensbasierte Systeme

- Multikriterielle Analyse

- Optimierungssysteme und -anwendungen

- Risikoanalyse

- Robustheit und Sensitivitätsanalyse

- Textanalyse/Text-Mining

- Modellierung von Unsicherheit

- Web Analytics

\section{Einreichung von Beiträgen}

Bitte reichen Sie Beiträge für das Schwerpunktheft bis spätestens 2013-07-01 über das Online-Begutachtungssystem (http:// www.editorialmanager.com/buis/) der Zeitschrift unter der Rubrik „BISE Decision Analytics" ein. Bitte beachten Sie die Hinweise zu formaler Gestaltung und Umfang von Beiträgen für die WIRTSCHAFTSINFORMATIK/Business \& Information Systems Engineering (BISE). Vollständige Beiträge sollten höchstens 
50.000 Zeichen einschließlich Leerzeichen umfassen, abzüglich 5.000 Zeichen je Seite an Bildern. Ausführliche Autorenrichtlinien stehen unter http://www.wirtschaftsinformatik.de zum Download bereit.

Eingereichte Beiträge werden anonymisiert von mehreren Fachgutachtern in einem doppelt-blinden Verfahren auf Relevanz, Originalität und fachliche Qualität beurteilt.

\section{Zeitplan}

Einreichung von Beiträgen bis: 2013-07-01
Benachrichtigung der Autoren: 2013-08-26

Abschluss der Überarbeitung: 2013-10-28

Benachrichtigung der Autoren: 2013-12-16

Ggf. Abschluss einer zweiten Überarbeitung: 2014-01-20

Geplanter Erscheinungstermin des Heftes: Juni 2014 